\title{
P04-48. HIV-I envelope induces memory B cell responses that correlate with plasma antibody levels after gp I 20 protein vaccination or chronic HIV-I infection
}

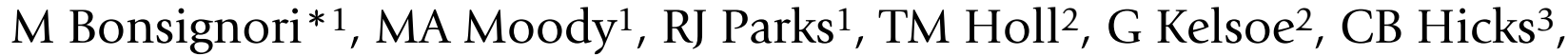 \\ $\mathrm{N}$ Vandergrift ${ }^{1}$, GD Tomaras ${ }^{1}$ and BF Haynes ${ }^{1}$
}

Address: ${ }^{1}$ Department of Medicine, Duke University Medical Center - Duke Human Vaccine Institute, Durham, NC, USA, ${ }^{2}$ Duke University Medical Center - Department of Immunology, Durham, USA and ${ }^{3}$ Duke University Medical Center - Department of Medicine, Durham, USA

* Corresponding author

from AIDS Vaccine 2009

Paris, France. 19-22 October 2009

Published: 22 October 2009

Retrovirology 2009, 6(Suppl 3):P76 doi:10.1186/1742-4690-6-S3-P76

This abstract is available from: http://www.retrovirology.com/content/6/S3/P76

(c) 2009 Bonsignori et al; licensee BioMed Central Ltd.

\section{Background}

Successful vaccines (e.g. tetanus) can induce long-lived antibody levels that are maintained by long-lived plasma cells and do not correlate with numbers of blood memory B-cells. Early events during HIV-1 acute infection may impair the timely onset of neutralizing antibody responses. Thus, an effective HIV-1 vaccine should elicit high levels of durable antibodies by long-lived plasma cells. We asked if HIV-1 envelope-specific memory responses are sustained by memory B-cells in the settings of HIV-1 gp120 envelope vaccination and chronic HIV-1 infection (CHI).

\section{Methods}

Total, gp140 envelope and V3-specific IgG memory B-cells from PBMCs of $26 \mathrm{CHI}$ patients and 25 vaccinated volunteers from the VaxGen clinical trial VAX004 were enumerated with ELISpot assays after in vitro stimulation. Respective plasma antibody levels were tested with ELISA. An additional $8 \mathrm{CHI}$ subjects, treated with ART for between 125 and 387 weeks and with viremia suppression, were studied for levels of anti-gp120, -gp41, -p55, tetanus toxoid and -influenza IgG plasma antibodies (Luminex assay and ELISA) over time of ART to determine the relative antibody level half-lives.

\section{Results}

Levels of anti-HIV-1 envelope plasma antibodies and memory B-cells correlated both in $\mathrm{CHI}$ and vaccinated individuals. Moreover, whereas the reported expected half-life of plasma antibody levels to protein vaccines is $>10$ years when maintained by long-lived plasma cells (we observed $~ 11$ years for tetanus), plasma anti-envelope antibody level half-lives were 33-81 weeks in ARTinduced aviremic $\mathrm{CHI}$ subjects. In contrast, anti-p55 Gag antibody level half-life was 648 weeks and antibody titers against influenza did not decay in-between yearly or biennial influenza vaccine boosts.

\section{Conclusion}

These data demonstrated that HIV-1 envelope induces predominantly short-lived memory B-cell-dependent plasma antibodies in the settings of envelope vaccination and chronic HIV-1 infection. The inability to generate high titers of long-lived anti-envelope antibodies is a major hurdle to overcome for the development of a successful HIV-1 vaccine. 